15

\title{
Повышение информативности аэробаллистического эксперимента как мера повышения точности определения коэффрициента сопротивления
}

\author{
(C) С.И. Герасимов, ${ }^{1,2,3,4,5}$ В.А. Кикеев, ${ }^{2,3}$ А.П. Фомкин, ${ }^{1}$ \\ ${ }^{1}$ Российский федеральный ядерный центр - \\ Всероссийский научно-исследовательский институт экспериментальной фризики, \\ 607190 Саров, Россия \\ 2 Институт проблем машиностроения РАН, \\ 603024 Нижний Новгород, Россия \\ ${ }^{3}$ Нижегородский государственный технический университет им. Р.Е. Алексеева, \\ 603950 Нижный Новогород, Россия \\ ${ }^{4}$ Саровский ффизико-технический институт НИЯУ МИФИ, \\ 607186 Саров, Россия \\ 5 Национальный исследовательский ядерный университет „МИФИ“, \\ 115409 Москва, Россия \\ e-mail: s.i.gerasimov@mail.ru
}

(Поступило в Редакцию 16 февраля 2016 г.)

Проведен анализ оценки погрешности определения коэффициента сопротивления по результатам проведения аэробаллистического эксперимента. Предложены технические решения, схемы и устройства, позволяющие повысить информативность опыта и, в конечном итоге, уменьшить погрешность определения коэффициента сопротивления.

\section{Введение}

Одной из традиционных задач экспериментальной баллистики является определение аэродинамических характеристик объекта испытаний по его траекторным данным, полученным в аэробаллистическом эксперименте (АБЭ). Именно эта задача предъявляет жесткие требования к точности регистрирующего оборудования и числу постов регистрации. Известно, что определение аэродинамических сил и моментов в линейной форме через динамические переменные требует использования 60 устойчивых производных, однако наличие симметрии сведет их число к 11-ти различным производным, не равным нулю. Поскольку симметричный снаряд часто имеет большую скорость вращения, обычно рассматриваются члены второго порядка, содержащие угловую скорость вращения, выраженные в форме Магнуса. Для случая симметрии имеется лишь восемь различных выражений этого типа. Из общего числа 19 коэффициентов $(11+8)$ могут потребоваться лишь семь, если считать только те коэффициенты, которые оказывают соизмеримое влияние на движение. Таким образом, даже в простейшем случае осесимметричного вращающегося снаряда необходимо иметь как минимум семь постов регистрации, чтобы определить необходимые производные аэродинамических коэффициентов с нулевым числом степеней свободы (режим коллокации, аналогичный интерполяции, в отличие от аппроксимации при наличии степеней свободы). Ограничением числа постов регистрации сверху служат обычно условия, внешние по отношению к решаемой задаче, среди которых сле- дует назвать стоимость, производственные площади, трудоемкость эксплуатации и обработки получаемых материалов, наконец, просто здравый смысл, опирающийся на статистические оценки точности получаемых результатов.

От качества аэродинамической отработки, т. е. от того, насколько точно определены аэродинамические характеристики (АДХ) объекта испытания (ОИ) и в первую очередь коэффициент силы лобового сопротивления $C_{x}$, зависит точность прогнозирования функционирования реального летательного аппарата (ЛА) во всех условиях его применения.

Аэробаллистический метод определения АДХ широко распространен в отечественной и мировой практике (в мире существуют более сотни аэробаллистических установок) и используется достаточно давно. Аэробаллистические испытания с целью определения лобового аэродинамического сопротивления артиллерийских снарядов проводились еще в середине XIX века.

Имеющиеся данные по случайной среднеквадратической погрешности значений, определяемых аэробаллистическим методом, обладают достаточной полнотой лишь в частном случае, когда $C_{x}=$ const. Имеет смысл оценить погрешность определения нелинейного $C_{x}(M)$ для оптимального планирования аэробаллистического эксперимента.

Суть эксперимента в аэробаллистическом тире (АБТ) состоит в следующем. Метательной установкой, пороховой или легкогазовой, баллистическая модель, геометрически подобная отрабатываемому ЛА, разгоняется до заданной скорости и пролетает измерительный участок 
тира, где средствами фотограмметрии осуществляются внешнетраекторные измерения линейных координат центра масс модели $X$ в последовательные моменты времени $t_{i}$. Полученная последовательность значений $X$ отражает фактическую зависимость $X\left(t_{i}\right)$, являющуюся вторым интегралом от действующей на модель в полете силы лобового сопротивления и поэтому содержит информацию, на основе которой может быть определена зависимость $C_{x}(M)$ в опыте. Для определения $C_{x}(M)$ необходимо также знать конструктивные параметры модели - диаметр миделя $\varnothing$, вес $G$, метеоусловия в момент проведения опыта: давление $P$ и температура $T$ газа на измерительном участке - и определить начальные условия опыта в некоторой произвольной точке траектории полета в момент $t_{0}$ : скорость $V_{0}$ и координату $x_{0}$ центра масс модели.

Точность определения $C_{x}$ по материалам эксперимента в АБТ зависит от точности определения зависимости $X\left(t_{i}\right)$, конструктивных параметров модели и метеоизмерений. При этом нужно учесть, что если часть параметров, включая вес модели и ее обводы, а также температуру, влажность, давление на измерительном участке АБТ, можно определить с высокой точностью перед проведением опыта, то гарантий, что, например, после выхода из ствола модель сохранит обводы и начальное состояние маркированной поверхности, а также реализует заданные начальные условия по скорости и углу атаки, нет, и при планировании эксперимента необходимо помимо технических решений по сохранению параметров модели в опыте предусмотреть дополнительные схемы контроля в процессе опыта и разработать соответствующие устройства запуска и регистрации.

\section{Оценка точности при постоянном $C_{x}$}

Для относительно малых измерительных участков, где замедление скорости движения незначительно и где искомый коэффициент сопротивления может считаться постоянным, закон изменения координаты по времени определяется как [1]

$$
x=\frac{2 G}{\rho S g C_{x}} \ln \left[\frac{\rho S g C_{x}}{2 G} V_{0} t+1\right],
$$

где $G, S$ - параметры модели, $\rho$ - параметр среды, $V_{0}$ - начальная скорость в земной системе координат в момент времени $t_{0}$. В случае движения модели в баротрассе или аэродинамической трубе при наличии встречного равномерно движущегося потока со скоростью $V_{n}$, член $V_{0} t$ в (1) заменяется на $\left(V_{0}+V_{n}\right) t$ и добавляется слагаемое $-V_{n} t$. Из выражения (1) следует, что даже в простейшем случае движения, когда $C_{x}=\mathrm{const}, C_{x}$ в точном виде явно не определяется. Приближенное выражение для $C_{x}$ рассматривается как функция независимых параметров, получаемых в эксперименте. $C_{x}=\frac{4 G}{\rho S g} \frac{V_{0} t-x}{V_{0}^{2} t^{2}}$, или в случае фиксации моментов времени $t_{1}, t_{2}$ в сечениях $x_{1}, x_{2}$

$$
\left(x_{0}<x_{1}<x_{2}\right) C_{x}=-\frac{4 G}{\rho S g} \frac{\left(t_{2} / t_{1}\right) x_{1}-x_{2}}{\left(t_{2} / t_{1}\right) x_{1}^{2}-x_{2}^{2}},
$$

при наличии встречного потока знаменатель второго сомножителя в (2) выглядит как

$$
\frac{t_{2}}{t_{1}}\left[\left(x_{1}+V_{n} t_{2}\right)^{2}-\left(x_{2}+V_{n} t_{2}\right)^{2}\right] .
$$

Взяв приращение по различным параметрам формулы (2), определим полную относительную ошибку коэффициента сопротивления

$$
\begin{aligned}
& \frac{d C_{x}}{C_{x}}=\delta \bar{C}_{x}= \pm\left[\frac{1}{G} \Delta G+\frac{1}{\rho} \Delta \rho+\frac{2}{r} \Delta r+\frac{2}{V_{0}} \Delta V_{0}\right. \\
& \left.+\frac{2}{x_{1} / x_{2}\left(1-x_{1} / x_{2}\right)} \frac{1}{l} \Delta x_{1}+\frac{2 V_{0}}{x_{1} / x_{2}\left(1-x_{1} / x_{2}\right)} \frac{1}{l} \Delta t_{1}\right]
\end{aligned}
$$

(множитель 2 в выражениях ошибок по времени и расстоянию получается из-за двукратного измерения базы $\left(x_{2}, x_{1}\right)$ и времени, $l$ - декремент расстояния, определяемый из отставания модели, испытывающей сопротивление, по расстоянию за время $t$ от модели, движущейся с постоянной начальной скоростью: $l=\frac{1}{2} \frac{\rho S C_{x} g}{G} x^{2}\left(1+\frac{V_{n}}{V_{0}}\right)^{2}$. Анализ данной зависимости показывает, что те измерения, которые проводятся до опыта, например взвешивание модели, желательно проводить с точностью до десятых долей процента. Другие ошибки, зависящие от величины замедления и конструктивных размеров, для своего уменьшения требуют варьирования, например, длиной измерительной трассы или начальными условиями на баротрассе (статическое давление).

\section{Анализ источников и вида погрешностей определения коэффициента $C_{x}(M)$ в аэробаллистическом эксперименте}

Коэффициент сопротивления в связной системе координат при нулевом угле атаки $C_{x}=C_{\tau_{0}}=\frac{2 G}{\rho S g} \ddot{\ddot{X}}$ определяется в результате согласования решения системы дифференциальных уравнений движения с внешнетраекторными измерениями линейных и угловых координат $X\left(t_{i}\right)$ в опыте. Поэтому на точность определения $C_{x}$ влияют как погрешности измерений $X\left(t_{i}\right)$, так и погрешности измерения констант, входящих в систему дифференциальных уравнений движения. Также факторами погрешности являются приводящие к нарушению геометрического подобия малые искажения обводов баллистических моделей, обусловленные технологическими допусками на их изготовление и возможными воздействиями на стадии внутренней баллистики, и возможная погрешность 
Таблица 1. Общая сводка погрешностей измерений

\begin{tabular}{|c|c|c|c|c|}
\hline \multirow{3}{*}{$\begin{array}{c}\text { Измеряемые } \\
\text { величины }\end{array}$} & \multirow{3}{*}{$\begin{array}{c}\text { Источники } \\
\text { погрешности }\end{array}$} & \multicolumn{3}{|c|}{ Вид погрешности } \\
\hline & & \multirow{2}{*}{$\begin{array}{c}\text { случайная } \\
\sigma\end{array}$} & \multicolumn{2}{|c|}{ систематическая } \\
\hline & & & $\delta 1$-типа & $\delta$ 2-типа \\
\hline$X\left(t_{i}\right)$ & $\begin{array}{l}\text { Разнообразные } \\
\text { юстировочные работы } \\
\text { Нестабильность } \\
\text { маркировки моделей }\end{array}$ & $\begin{array}{c}1-2.6 \mathrm{~mm} \\
- \\
- \\
-\end{array}$ & $\begin{array}{c}- \\
0.6 \mathrm{~mm} \\
0.3 \mathrm{~mm} \\
-\end{array}$ & $\begin{array}{c}- \\
- \\
- \\
0.6 \mathrm{~mm}\end{array}$ \\
\hline$D$ & $\begin{array}{l}\text { Субъективный } \\
\text { Установка нуля }\end{array}$ & $\begin{array}{c}0.01 \mathrm{~mm} \\
-\end{array}$ & $\begin{array}{c}- \\
\text { Пренебрежима мала }\end{array}$ & - \\
\hline $\begin{array}{l}G \\
T\end{array}$ & $\begin{array}{l}\text { Субъективный } \\
\text { Тарировка } \\
\text { Субъективный } \\
\text { Тарировка }\end{array}$ & $\begin{array}{c}0.02-0.1 \mathrm{~g} \\
- \\
0.4^{\circ} \mathrm{C} \\
-\end{array}$ & $\begin{array}{c}- \\
0.03 \mathrm{~g} \\
- \\
0.3^{\circ} \mathrm{C}\end{array}$ & $\begin{array}{l}- \\
- \\
- \\
-\end{array}$ \\
\hline$P$ & $\begin{array}{l}\text { Субъективный } \\
\text { Тарировка }\end{array}$ & $\begin{array}{c}0.4 \mathrm{~mm} \mathrm{Hg} \\
-\end{array}$ & $\begin{array}{c}- \\
0.4 \mathrm{~mm} \mathrm{Hg}\end{array}$ & - \\
\hline$R$ & Изготовление модели & - & $0.1 \mathrm{~mm}$ & - \\
\hline
\end{tabular}

математической модели эксперимента, используемой в алгоритме его обработки.

Существуют два вида погрешностей исходных данных: случайные независимые погрешности измерений, например, распределенные по нормальному закону, и случайные зависимые погрешности с неопределенным законом распределения. Первую погрешность назовем случайной, вторую - систематической. Методическая погрешность рассматривается как систематическая, обусловленная возможной неточностью методики обработки материалов АБЭ с целью определения $C_{x}$. Наличие систематических погрешностей вносит серьезные дополнительные трудности в анализ точности. Во-первых, их непросто обнаружить, так как они во многих случаях не проявляют себя в разбросе экспериментальных данных, a, во-вторых, для их строгого учета необходимо знание корреляционной функции ошибок измерений, зависящей, причем, возможно, сложным образом, от многих физических факторов.

Случайные и систематические составляющие погрешностей измерений рассматриваются независимо.

Анализ случайных погрешностей осуществляется, исходя из нормального закона их распределения и с использованием среднеквадратических оценок $(\sigma)$, при этом погрешность нелинейной функции случайных величин оценивается посредством ее линеаризации в области распределения погрешностей аргументов.

Анализ систематической погрешности осуществляется в предельной постановке: оцениваются прямыми и косвенными методами максимально возможные величины систематических погрешностей $\delta$ без учета фактического вида их распределения, затем на тестах выявляются наиболее неблагоприятные с точки зрения точности $C_{x}$ виды систематической погрешности и в предположении наихудшего случая, что именно такие виды имеют фактические систематические погрешности, характеризуемые известной предельной величиной $\delta$, производится оценка их составляющей в погрешности $C_{x}$. Такая оценка является оценкой сверху, она гарантирует, что реальные предельные систематические составляющие погрешности коэффициента $C_{x}$ не превышают полученных величин.

В табл. 1 дана общая сводка проанализированных итоговых значимых погрешностей измерений.

Линеаризуем выражение для $C_{x}$ и, согласно правилам оценки среднеквадратической и предельной погрешностей, получим

$$
\begin{gathered}
\sigma^{2} C_{x}=\sigma_{G}^{2} C_{x}+\sigma_{D}^{2} C_{x}+\sigma_{P}^{2} C_{x}+\sigma_{x}^{2} C_{x}, \\
\delta C_{x}=\delta_{G} C_{x}+\delta_{D} C_{x}+\delta_{P} C_{x}+\delta_{x} C_{x}+\delta_{M} C_{x}, \\
\sigma_{G} C_{x}=\frac{C_{x}}{G} \sigma_{G}, \quad \sigma_{D} C_{x}=\frac{2 C_{x}}{D} \sigma_{D}, \quad \sigma_{p} C_{x}=\frac{C_{x}}{p} \sigma_{p}, \\
\sigma_{T} C_{x}=\frac{C_{x}}{T+273.15} \sigma_{T}, \quad \delta_{G} C_{x}=\frac{C_{x}}{G} \delta_{G}, \\
\delta_{D} C_{x}=\frac{2 C_{x}}{D} \delta_{D}, \quad \delta_{p} C_{x}=\frac{C_{x}}{p} \delta_{p}, \quad \delta_{T} C_{x}=\frac{C_{x}}{T+273.15} \delta_{T} .
\end{gathered}
$$

Суммарную предельную погрешность $\delta_{\Sigma} C_{x}$ определим как

$$
\delta_{\Sigma} C_{x}=2 \sigma C_{x}+\delta C_{x} .
$$

Проведем расчетный анализ случайной погрешности $\sigma_{x} C_{x}$, обусловленной случайными ошибками измерений $X\left(t_{i}\right)$, используя методику математической обработки эксперимента, изложенную в работе [2]. Представляя $C_{x}(M)$ в виде квадратичной трехпараметрической 
зависимости, имеем следующее уравнение движения для анализа:

$$
\begin{aligned}
\dot{V}= & C_{x}(M) \frac{\rho S g}{2 G} V^{2}=\left[C_{x 0}\left(V / a_{0}\right)+a_{1}\right. \\
& \left.+a_{2}\left(V / a_{0}-M_{0}\right)+a_{3}\left(V / a_{0}-M_{0}\right)^{2}\right] \frac{\rho S g}{2 G} V^{2},
\end{aligned}
$$

где $C_{x 0}(M)$ - априорная зависимость $C_{x}(M)$, полученная расчетно или из тестовых юстировочных испытаний; $a_{0}-$ скорость звука; $a_{1}, a_{2}, a_{3}$ - параметры, определяемые по результатам опыта. При $a_{3}=0$ имеем двухпараметрическую зависимость, а при $a_{2}=a_{3}=0$ однопараметрическую зависимость для $C_{x}(M)$ (практическое значение имеют только виды, включающие $a_{1}$ ).

C учетом итерационного метода решения уравнений движения численно среднеквадратическая погрешность определяется из формулы

$$
\begin{aligned}
\sigma_{x}^{2} C_{x}= & \sigma^{2} a_{1}+\sigma^{2} a_{2}\left(M-M_{0}\right)^{2}+\sigma^{2} a_{3}\left(M-M_{0}\right)^{4} \\
& +2 \sigma^{2} a_{12}\left(M-M_{0}\right)+2 \sigma^{2} a_{13}\left(M-M_{0}\right)^{2} \\
& +2 \sigma^{2} a_{23}\left(M-M_{0}\right)^{2},
\end{aligned}
$$

где $\mathbf{a}^{T}=\left(a_{1}, a_{2}, a_{3}, X_{0}, V_{0}\right)$ - вектор параметров согласования, используемый при минимизации функционала [2]:

$$
\Phi^{(v)}=\frac{1}{\sigma^{2} X\left(N-N_{a}\right)} \sum_{i=1}^{N}\left[X\left(\mathbf{a}^{-v}, t_{i}\right)-X\left(t_{i}\right)\right]^{2},
$$

где $-\sigma X-$ случайная среднеквадратическая погрешность определения зависимости $X\left(t_{i}\right), N_{a}$ - количество параметров согласования, $v$ - порядковый номер итерации, $X\left(\mathbf{a}^{(v)}, t_{i}\right)$ - решение системы уравнений движения. Зависимость $C_{x}(M)$ определена параметрами $a_{1}, a_{2}, a_{3}$. Если известна дисперсионная матрица е параметров, то $C_{x}(M)$ можно вычислить по формуле (7). Для расчета $\mathbf{e}$ используется формула

$$
\Delta \mathbf{a}^{(v)}=\left(\tilde{e}^{(v)}\right) \mathbf{B}^{(v)},
$$

где

$$
\begin{gathered}
\tilde{e}^{-1_{(v)}}=\frac{1}{\sigma^{2} X} \sum_{i=1}^{N} \mathbf{H}_{i}^{T_{(v)}} ; \\
\mathbf{B}^{(v)}=\frac{1}{\sigma^{2} X} \sum_{i=1}^{N} \mathbf{H}_{i}^{T_{(v)}}\left[X\left(\mathbf{a}^{(v)}, t\right)-X\left(t_{i}\right)\right],
\end{gathered}
$$

$\mathbf{H}_{i}$ - вектор частных производных расчетных значений координаты $X$ по параметрам согласования $a_{j}$. Элементы вектора $\mathbf{H}_{i}$ определяются численным дифференцированием по формуле

$$
H_{i}^{j}=\frac{X\left(a_{1}, a_{2}, \ldots, a_{j}+\Delta a_{j}, \ldots, V_{0}, t_{i}\right)-}{-X\left(a_{1}, a_{2}, \ldots, a_{j}, \ldots, V_{0}, t_{i}\right)} .
$$

Формула (9) получена на основе линеаризации устанавливаемых системой уравнений движения и процедуры минимизации функционала $\Phi$ зависимостей параметров $a_{1}, a_{2}, a_{3}$ от информации опыта $X: a_{j}=a_{j}\left(X_{i}\right)$,

$$
\Delta a_{j}=\sum_{i=1}^{N} \frac{\partial a_{j}}{\partial X_{i}} \Delta X_{i}
$$

где $\Delta a_{j}, \Delta X_{i}$ - приращение $a_{j}$ и расчетных значений $X_{i}$ на конечной итерации, когда различие между расчетами и опытными значениями $X_{i}$ обусловлены только ошибками $\sigma X$. Производные $\frac{\partial a_{j}}{\partial X_{i}}$ определяются через обратные производные $\frac{\partial X_{i}}{\partial a_{j}}$, вычисляемые численным методом. Полученные таким образом оценки $\sigma C_{x}$ справедливы, если линеаризация функций $a_{j}\left(X_{i}\right)$ дает их хорошее приближение в области $\left|\Delta X_{i}\right|=3 \sigma X$, что не является очевидным и требует проверки. В тестах при различных реализациях возмущений $X_{i}$ вычислены отклонения $\Delta a_{1}, \Delta a_{2}, \Delta a_{3}$ и оценки погрешностей $\delta a_{1}$, $\delta a_{2}, \delta a_{3}$. В нормальном случае оценки среднеквадратической погрешности $\sigma \Delta a_{1}, \sigma \Delta a_{2}, \sigma \Delta a_{3}$, вычисленные по разбросу

$$
\sigma \Delta a_{j}=\frac{\sqrt{\sum_{i=1}^{N_{i}} \Delta a_{j i}}}{N_{1}-1},
$$

должны быть близки к соответствующим оценкам $\sigma a_{j}$. Здесь $i-$ порядковый номер теста, $N_{i}$ - количество тестов.

Если воспользоваться только численным подходом для анализа $\sigma C_{x}$, во всей области условий АБЭ, то потребуется большое количество вариантов расчета, чтобы выявить влияние на $\sigma C_{x}$ тех или иных факторов. Поэтому крайне желательно получение приближенных аналитических формул, определяющих зависимость $\sigma C_{x}$ от условий АБЭ. Такие формулы желательно получить для постоянного $\left(C_{x_{0}}(M)=a_{1}\right)$, квадратичного и линейного вида зависимости $C_{x}$. Если принять упрощенную схему опыта, при которой аэродинамическая сила сопротивления постоянна в отдельном опыте и прямопропорциональна квадрату скорости в середине траектории $\left(\frac{\rho S g}{2 G} C_{\tau 0} V_{0}^{2}\right)$, то решение уравнения движения при $t_{0}=0$ в этом случае будет следующим:

$$
X=X_{0}+V_{0} t+\frac{V_{0}^{2} \rho S g}{2 G} t^{2} .
$$

Для случая обработки отдельного опыта, когда по завершении процесса итерации вычисляется дисперсионная матрица $\mathbf{e}=\Phi \mathbf{e}_{1}$, определяется среднеквадратическая погрешность параметров согласования

$$
\sigma a_{j}=\sqrt{\Phi e_{j j}}
$$

Пользуясь формулой (11) с учетом зависимости (12) имеем следующие значения производных: $\frac{\partial X}{\partial X_{0}}=1$, 
$\frac{\partial V}{\partial V_{0}}=t+\frac{\rho S g}{2 G} V_{0} C_{x} t^{2} ; \frac{\partial X}{\partial C_{x}}=\frac{\rho S g}{2 G} V_{0}^{2} t^{2}$. Матрица Н имеет вид

$$
H=\left|\begin{array}{ccc}
N, & \sum\left(t_{i}+v_{0} k C_{x} t_{i}^{2}\right), & \left.\sum \frac{v_{0}^{2} k}{2} t_{i}^{2}\right) \\
\sum\left(t_{i}+v_{0} k C_{x} t_{i}^{2}\right), & \sum\left(t_{i}+v_{0} k C_{x} t_{i}^{2}\right)^{2}, & \sum\left(t_{i}+v_{0} k C_{x} t_{i}^{2}\right) \frac{v_{0}^{2} k}{2} t_{i}^{2} \\
\sum \frac{v_{0}^{2} k}{2} t_{i}^{2}, & \sum\left(t_{i}+v_{0} k C_{x} t_{i}^{2}\right) \frac{v_{0}^{2} k}{2} t_{i}^{2}, & \sum \frac{v_{0}^{4} k^{2}}{4} t_{i}^{4}
\end{array}\right| i \ldots N
$$

Здесь $k=\rho S g / 2 G$. Примем, что $N-$ нечетное, регистрация в опыте ведется с постоянным шагом $\Delta t$, в середине траектории $i=\frac{N+1}{2}$ время $t_{i}$ обращается в нуль и в силу этого при выполнении суммирования в (13) члены, содержащие $t_{i}$ в нечетной степени, взаимоуничтожаются и выражение для $\mathbf{H}$ упростится. Элемент $H_{11}^{-1}$ обращенной матрицы $h^{-1}$ равен $\tilde{\sigma}^{2} c_{x}$. Выполнив обращение, упростив полученное выражение и приняв, что $\Delta t=\frac{L}{v_{0}(N-1)}$, получим

$\tilde{\sigma} C_{x}=\frac{\sigma X 2 G}{\rho S g L^{2}} \sqrt{\frac{760(N-1)^{3}+48\left(k c_{\tau 0} L\right)^{2}\left(N^{2}-4\right)(N-1)}{(N+1) N\left(N^{2}-4\right)}}$.

Легко показать, что для трасс длиной $L \leq 200 \mathrm{~m}$ влиянием $C_{x}$ на $\sigma C_{x}$ можно пренебречь, и формула (14) для большинства трасс преобразуется к виду

$$
\tilde{\sigma} c_{x}=\frac{\sigma X}{k L^{2}} \sqrt{\frac{760(N-1)^{3}}{(N+1) N\left(N^{2}-4\right)}} .
$$

В табл. 2 приведены сопоставления оценок $\sigma C_{x}$, полученных с помощью точной (12) и приближенной (15) формул, для стандартной трассы в широком диапазоне условий.

\section{Отбраковка аварийных экспериментов}

Проблема отнесения опытов к зачетным требует дополнительного контроля состояния модели после ее разгона средствами метания перед попаданием на измерительный участок. Для оценки состояния модели на этапе промежуточной баллистики разработаны оптикорентгенографические посты регистрации. На рис. 1 приведены силуэтные изображения моделей в некоторых опытах, полученных с помощью разработанного аппарата, позволяющего получать картины объектов при фокусном расстоянии до $1 \mathrm{~m}$ при использовании рентгеновской пленки чувствительностью не менее $900-1000 \mathrm{P}^{-1}$ и флуоресцентных усиливающих экранов [3].

На рис. 2 приведена схема разработанного комбинированного фотопоста [4]. На рис. 3, 4 показаны характерные результаты регистрации состояния испытываемых моделей до входа на измерительный участок аэробаллистической трассы.

На рис. 3 показан пример обнаружения повреждения поверхности модели после разгона в стволе баллистической установки по характерному скачку на цилиндрическом участке поверхности.

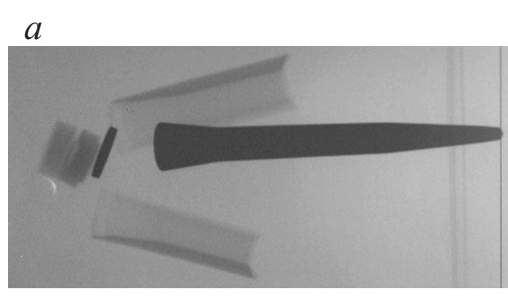

$b$
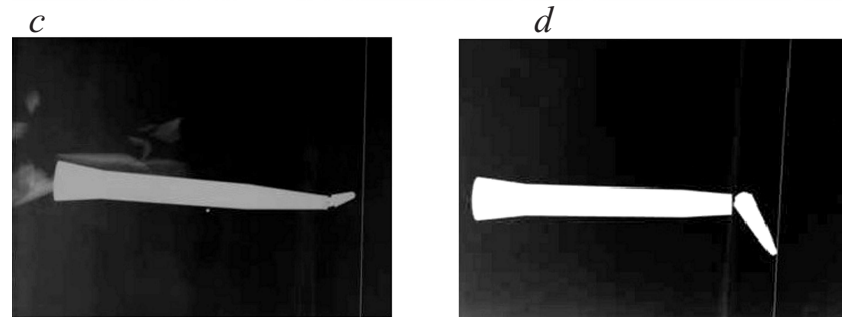

Рис. 1. Контроль состояния элементов поддона и их разлета при отделении от модели $(a)$ и характер разрушения модели в аварийных опытах $(b, c, d)$.
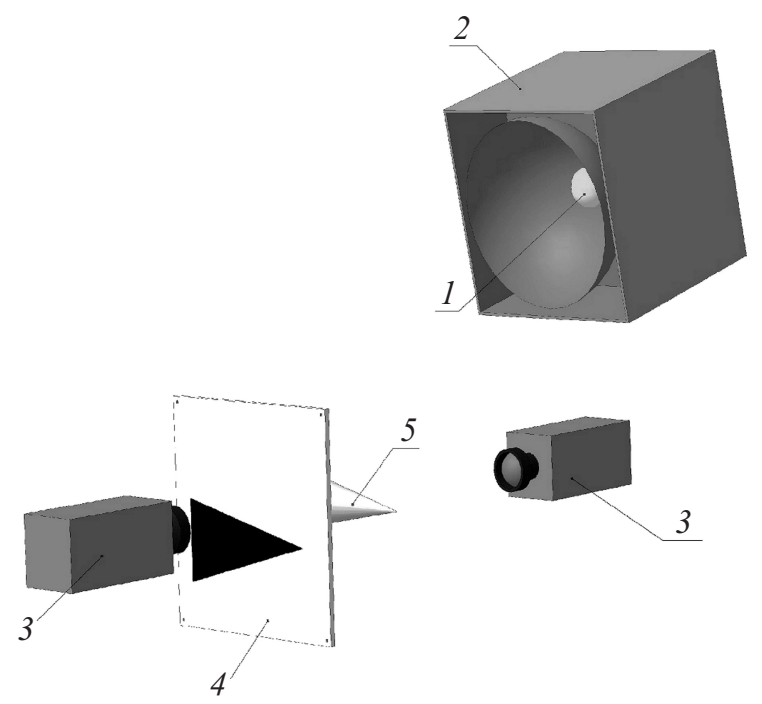

Рис. 2. Схема комбинированного поста для одновременного получения теневого изображения и фотоизображения в отраженном свете: 1 - точечный излучатель, 2 - газоразрядный накопитель, 3 - камера с затвором с ЭОП, 4 - полупрозрачный экран; 5 - регистрируемый объект исследования.

\section{Точное определение моментов построения изображений $t_{i}$}

Для точного определения моментов построения изображений при срабатывании импульсных источников све- 
Таблица 2. Сопоставление оценок $\sigma C_{x}$, полученных с помощью формул (12) и (15)

\begin{tabular}{c|c|c|c|c|c|c|c|c|c|c}
\hline Вид данных & Стандарт & $G=0.1$ & $G=2.5$ & $L=240$ & $L=60$ & $N=81$ & $N=11$ & $V=2028$ & $C_{\tau 0}=1.5$ & $C_{\tau 0}=0$ \\
\hline$\tilde{\sigma} C_{\text {хочнн }} \cdot 10^{-4}$ & 0.921 & 0.184 & 4.612 & 0.230 & 3.680 & 0.669 & 1.591 & 0.920 & 0.956 & 0.919 \\
\hline$\tilde{\sigma} C_{x} \cdot 10^{-4}$ & 0.926 & 0.187 & 4.621 & 0.232 & 3.690 & 0.684 & 1.586 & 0.926 & 0.962 & 0.924
\end{tabular}

Примечание. $\tilde{\sigma} C_{\tau 0 \text { точн }}-$ оценка, полученная по точной формуле $(12)$. Стандарт - типовые условия: $N=41, L=120 \mathrm{~m}, G=0.5 \mathrm{~kg}$, $D=0.08 \mathrm{~m}, C_{x}=0.3, V_{0}=1024 \mathrm{~m} / \mathrm{s}, \sigma X=1 \mathrm{~mm}$, в остальных столбцах отклонения условий от типовых.

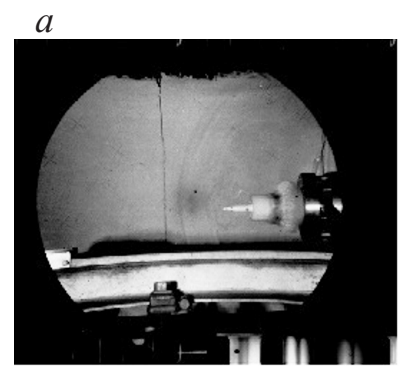

C
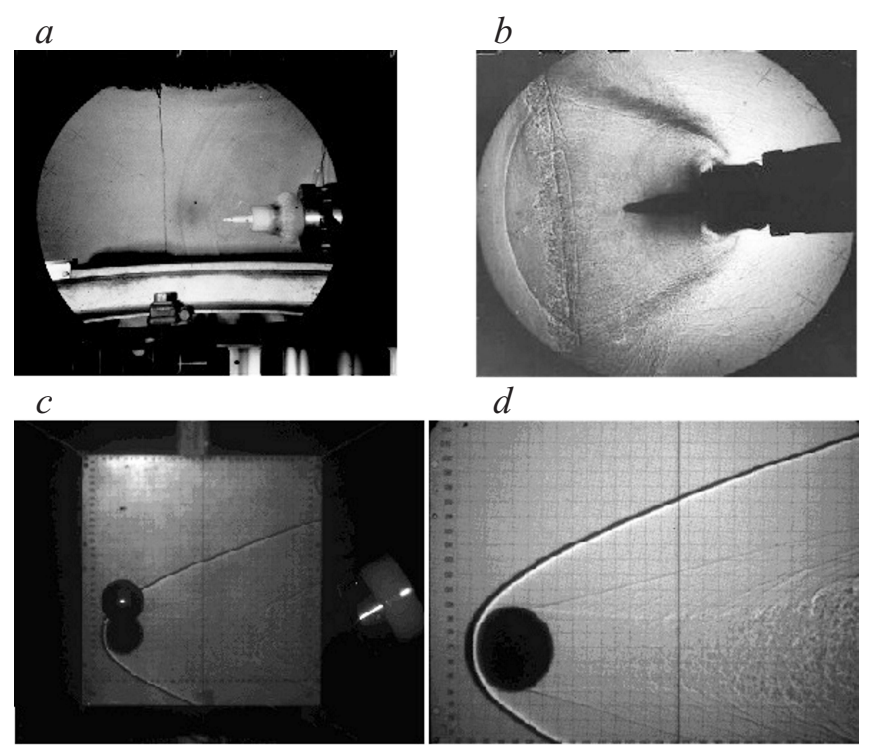

$d$

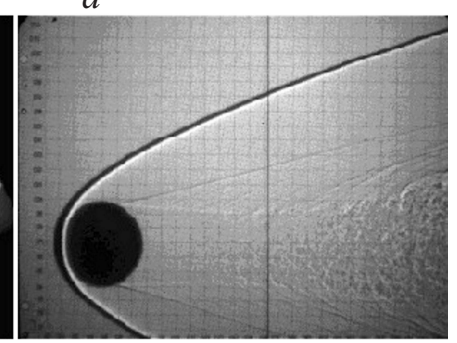

Рис. 3. Характерные примеры из баллистических экспериментов. Выход модели из канала ствола: снимок в отраженном свете $(a)$, теневая картина $(b)$; шар $(V=1472 \mathrm{M} / \mathrm{c})$ с отстающим поддоном: снимок в отраженном свете $(c)$, теневая картина $(d)$.

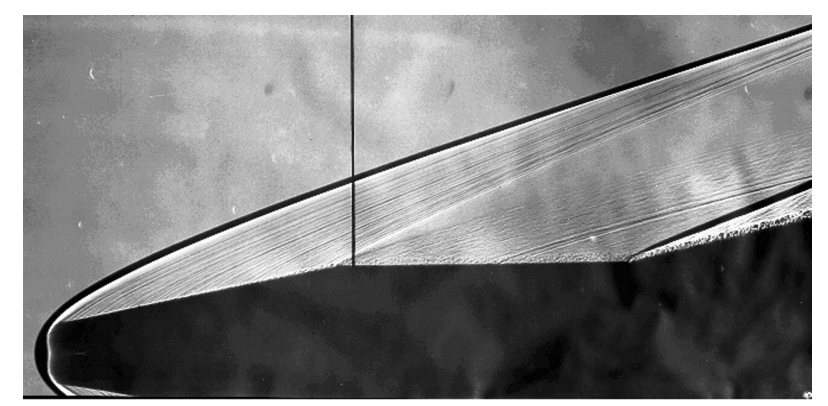

Рис. 4. Модель, получившая деформацию корпуса при разгоне в стволе баллистической установки.

та в аэробаллистическом эксперименте внедрен метод фотоэлектронного хронографирования последовательных энерговыделений в опыте [5]. Как показал анализ построения изображений, для привязки расшифрованных положений моделей $X_{i}$ к определенным моментам времени $t_{i}$ (хронографирование) требуется брать не момент запуска импульсного источника света, а время достижения амплитуды импульса. Таким образом требуется прописывать формы сигналов всех источников света $(N)$ в АБЭ. Используются датчики на базе кремниевых фотодиодов, включенных в фотодиодном режиме, при этом с точностью до $0.1 \mu \mathrm{m}$ фиксируется момент достижения амплитуды, который для разных источников может отличаться по нарастанию фронта в пределах $1-2 \mu \mathrm{s}$. Сюда же (погрешность, связанная со временем) отнесем требование к оптимальной длительности излучателя, строящего изображение в отраженном свете, поскольку камеры на измерительном участке трассы работают, как правило, в ждущем режиме (с открытым затвором), при увеличении времени экспонирования происходит смаз
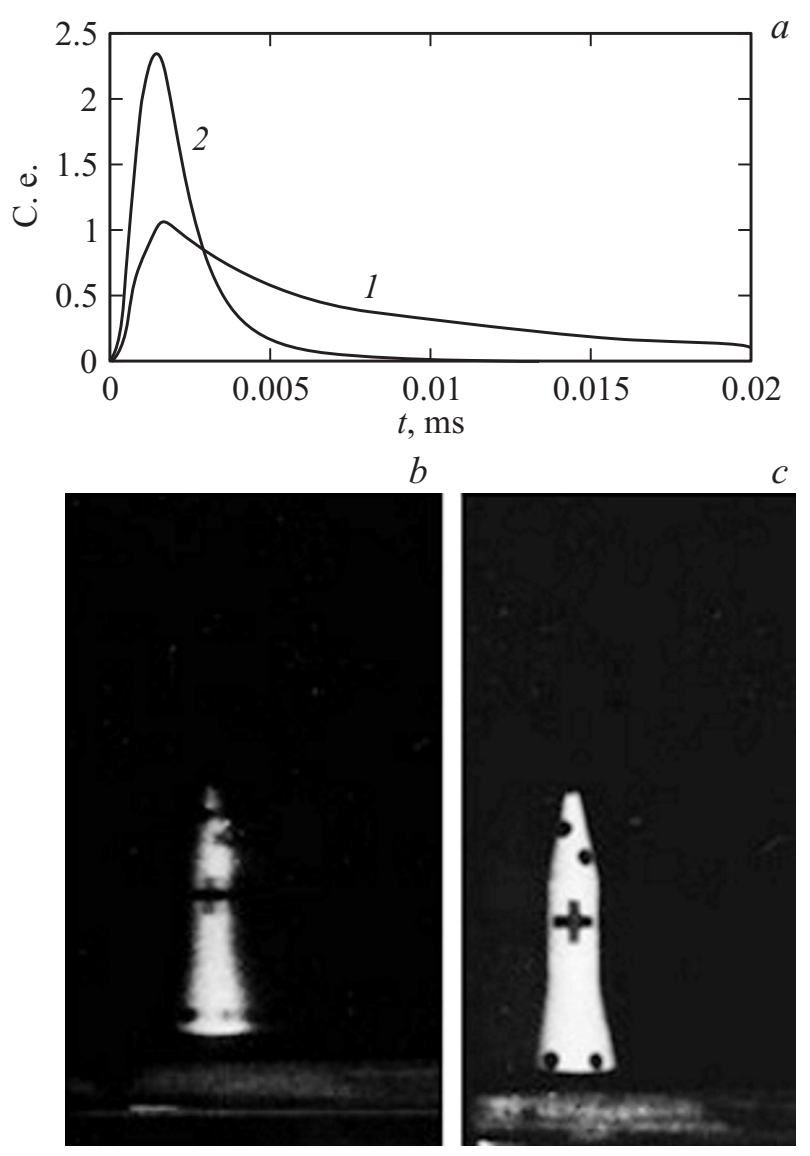

Рис. 5. Графики изменения освещенности во времени, создаваемой газоразрядными источниками: 1 - ИФК-500, 2 - ЭВИС (a). Сравнительные фотографии опыта при подсветке импульсным источником с лампой ИФК-500 (b) и ЭВИС (c). 


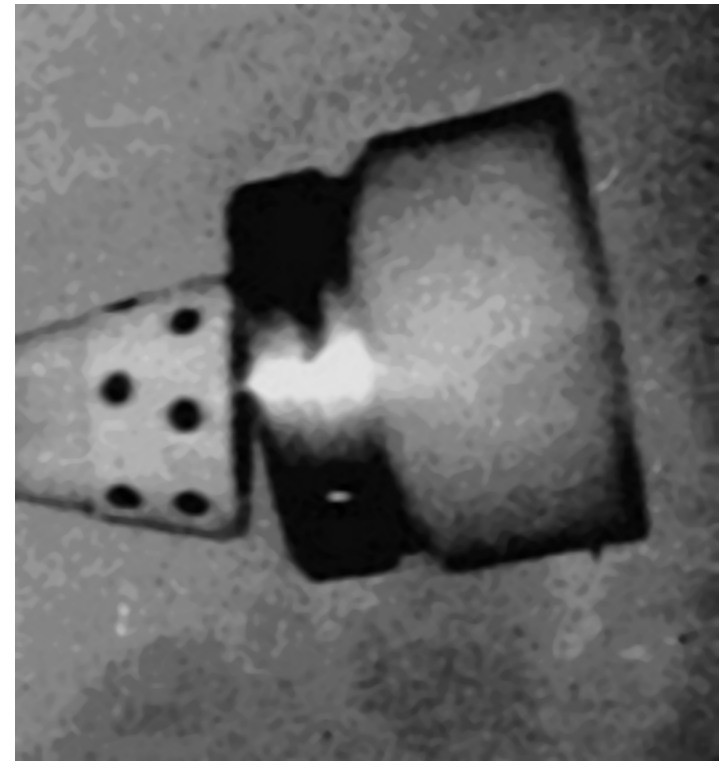

Рис. 6. Аэродинамическая модель после выхода из ствола. Дульная скорость $1740 \mathrm{~m} / \mathrm{s}$.

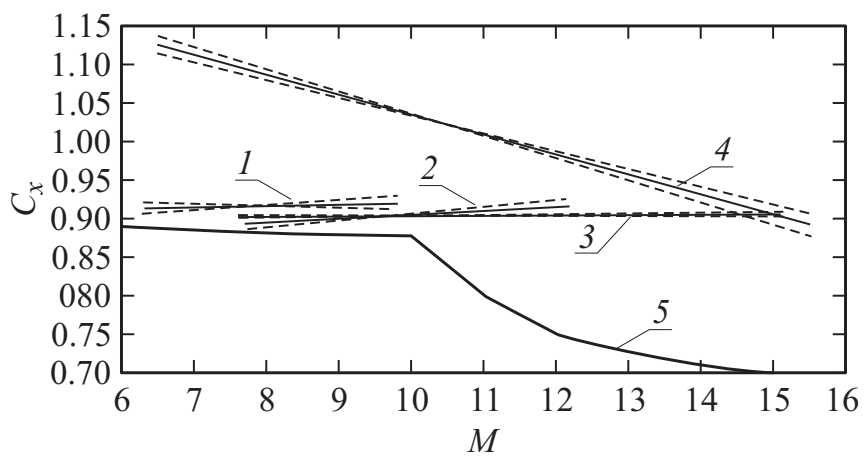

Рис. 7. Графики зависимости коэффициента силы лобового сопротивления $\left(C_{x}\right)$ от числа Маха $(M)$ для шаров из различных конструкционных материалов: 1 - опыт № $1,2-$ опыт № 2, 3 - опыт № 3, 4 - опыт № 4, 5 - по данным численного моделирования. изображения, что снижает точность расшифровки реперных знаков (марок) на поверхности модели. С учетом масштаба съемки и разрешения снимка для каждого скоростного режима существует предельное время выдержки, выше которого точность обработки, а следовательно, и определение коэффициента сопротивления упадет. Для режимов испытаний с $M \geq 4$ разработан излучатель ЭВИС [6], использующий расширение плазмы в зазоре между двумя незамкнутыми пластинами, что в отличие от замкнутых газоразрядных ламп типа ИФК, ИСП позволяет реализовать после окончания разряда „мгновенное“ остывание вместо длительного остывания у стенки диэлектрика (лампы).

На рис. 5 - сравнение работы двух излучателей от одного и того же накопителя энергии.

\section{Уменьшение тепловых нагрузок на поверхность разгоняемой модели}

Для снижения тепловых нагрузок на аэродинамическую модель при разгоне ствол баллистической установки вакуумируется и заполняется гелием [7]. С помощью разработанных оптических схем регистрации показан удовлетворительный результат при скоростях метания до $2.2 \mathrm{~km} / \mathrm{s}$ (рис. 6).

\section{Диапазон допустимых режимов юстировочных опытов с объектами простейших форм}

Аэробаллистические исследования тел простой формы используются в качестве тестовых объектов при юстировочных экспериментах на аэробаллистических трассах. В [8,9] показано, что сфера может быть использована в качестве тестового объекта вплоть до скорости $10 M$, когда численные значения хорошо совпадают с экспериментальными (рис. 7). При больших скоростях имеет место унос материала, проходящий по различным механизмам в зависимости от материала. На рис. 8

\section{$a$}

$b$

$c$

$d$

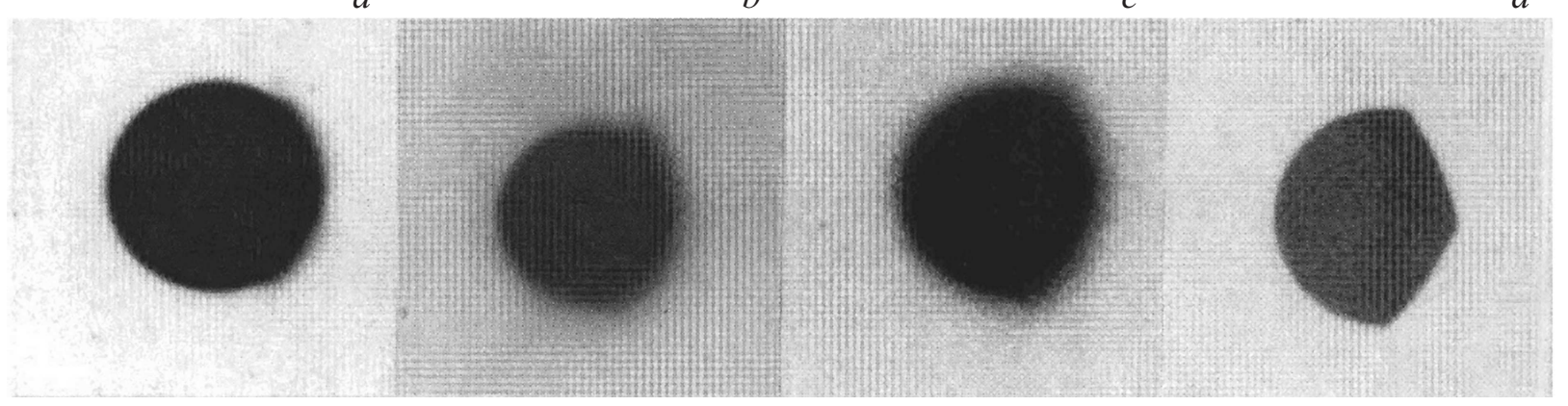

Рис. 8. Рентгеновские снимки шарика из композитного материала в различных стадиях полета: $a-X=14 \mathrm{~m}, V=4.9 \mathrm{~km} / \mathrm{s}, b-$ $X=26 \mathrm{~m}, V=4.6 \mathrm{~km} / \mathrm{s}, c-X=43 \mathrm{~m}, V=4.1 \mathrm{~km} / \mathrm{s}, d-X=156 \mathrm{~m}, V=1.5 \mathrm{~km} / \mathrm{s}$. 
(движение слева направо) показан характер разрушения композиционного материала с существенно различными температурами плавления компонентов.

\section{Заключение}

Проведен анализ влияния постановки и проведения аэробаллистического эксперимента на точность измерения аэродинамического коэффициента сопротивления. Выявлены наиболее значимые погрешности измерений и показаны методы повышения информативности эксперимента, позволяющие уменьшить эти погрешности.

Работа выполнена при поддержке Российского научного фонда, грант РНФ 16-38- 60125 мол_а_дк „Разработка методов повышения точности определения аэродинамических характеристик и внешнетракторных данных в аэробаллистических экспериментах“.

\section{Список литературы}

[1] Физико-газодинамические баллистические исследования. Сб. статей / Под ред. Г.И. Мишина. Л.: Наука, 1980.

[2] Осеева С.И., Викторов В.А., Неймаш Л.М., Садчиков Г.Д., Фотеев Ю.А. Идентификация аэродинамических параметров летательного аппарата по данным аэробаллистического эксперимента. Сб. тр. 3-й научной конф. Волжского регионального центра РАРАН „Современные методы проектирования и отработки ракетно-артиллерийского вооружения“. Саров: РФЯЦ-ВНИИЭФ, 2004. Т. 1. С. 214-221.

[3] Герасимов С.И., Полиенко Г.А., Хорошайло Е.С. Переносные импульсные рентгеновские аппараты серий „САРМА“ и „АРГУМЕНТ“ для регистрации быстропротекающих процессов. Саров, СарФТИ НИЯУ МИФИ, 2013. 65 с.

[4] Герасимов С.И., Ерофеев В.И., Сперанский А.В., Тотышев К.В. // ВАНТ. Серия ТПФ. 2013. В. 2. С. 40- 45.

[5] Герасимов С.И., Гончаров Е.А., Клунина Е.А. // Труды Межд. конф. 9-е Харитоновские тематические научные чтения „Экстремальные состояния вещества. Детонация. Ударные волны“. Саров, 2007. С. 708.

[6] Герасимов С.И., Файков Ю.И., Холин С.А. Кумулятивные источники света. Саров.: РФЯЦ-ВНИИЭФ, 2012. 326 с.

[7] Герасимов С.И., Клунина Е.А., Номаконова В.Н., Сальников А.В., Сперанский А.В. // Фундаментальные и прикладные проблемы техники и технологии. 2013. Вып. 4. C. 25-31.

[8] Герасимов С.И., Ерофеев В.И., Кикеев В.А., Фомкин А.П. // Вычислительная механика сплошных сред. 2013. Т. 6. Вып. 1. С. 34-41.

[9] Герасимов С.И., Лысенков В.Е., Осеева С.И., Тотышев К.В., Фомкин А.П. // ВАНТ, сер. ТПФ. 2012. В. 1. C. $52-63$. 